\title{
Inhibition of experimental asthma by indoleamine 2,3-dioxygenase
}

\author{
Tomoko Hayashi, ${ }^{1}$ Lucinda Beck, ${ }^{1}$ Cyprian Rossetto, ${ }^{1}$ Xing Gong, ${ }^{1}$ Osamu Takikawa, ${ }^{2}$ \\ Kenji Takabayashi, ${ }^{1}$ David H. Broide,1 Dennis A. Carson,1 and Eyal Raz'1
}

1 University of California, San Diego, Department of Medicine, La Jolla, California, USA. ${ }^{2}$ Central Research Institute, Graduate School of Medicine, Hokkaido University, Sapporo, Japan.

\begin{abstract}
Epidemiological evidence points to the inverse relationship between microbial exposure and the prevalence of allergic asthma and autoimmune diseases in Westernized countries. The molecular basis for this observation has not yet been completely delineated. Here we report that the administration of certain toll-like receptor (TLR) ligands, via the activation of innate immunity, induces high levels of indoleamine 2,3-dioxygenase (IDO), the rate-limiting enzyme of tryptophan catabolism in various organs. TLR9 ligand-induced pulmonary IDO activity inhibits Th2-driven experimental asthma. IDO activity expressed by resident lung cells rather than by pulmonary DCs suppressed lung inflammation and airway hyperreactivity. Our results provide a mechanistic insight into the various formulations of the hygiene hypothesis and underscore the notion that activation of innate immunity can inhibit adaptive Th cell responses.
\end{abstract}

\section{Introduction}

The dramatic increase in the prevalence of allergic and autoimmune diseases over the last 30 years suggests an important contributory role for environmental factors (1). The so-called hygiene hypothesis, first suggested by Strachan (2), proposes that a major source of microbial, Th1-like immune provocations has been lost with the decreased incidence of many infectious diseases due to vaccinations, the use of antibiotics, and other modern public health practices. The deficiency in Th1-like provocations leads to an increase in Th2-biased immune responses toward environmental allergens (3-6) and consequently to an increase in allergic asthma. While the evidence for a role of certain viral and bacterial exposure in protecting against allergic asthma is fairly strong $(7,8)$, the hygiene hypothesis in its Th1/Th2 formulation is unable to explain the protective role of helminth infection (a Th2-biased condition) in the development of allergic asthma (9). Furthermore, it also ignores the increase in the prevalence of Th1-mediated organ-specific autoimmune diseases that has been observed in affluent Westernized societies (10). These mechanistic inconsistencies of the hygiene hypothesis have been recently challenged by the "counter-regulation hypothesis." This hypothesis proposes that microbial infections induce regulatory $\mathrm{T}$ cell (Treg) responses, which display tolerogenic activities and therefore inhibit both Th2- and Th1-immune-mediated immunopathologies (10). Although the counter-regulation hypothesis provides an attractive explanation, the molecular pathways induced by microbial exposure and tolerogenic responses in the host have not yet been identified.

Nonstandard abbreviations used: airway hyperreactivity (AHR); 7-amino actinomycin D (7-AAD); bone marrow-derived DC (BMDC); enhanced pause (Penh); flagellin (Flag); IFN- $\alpha / \beta$ receptor (IFN- $\alpha / \beta$ R); IL-1 $\beta$ receptor (IL-1 $\beta R$ ); immunostimulatory sequence oligodeoxynucleotide (ISS-ODN); indoleamine 2,3 dioxygenase (IDO); kynurenine (KYN); methacholine (MCh); 1-methyl-DL-tryptophan (M-trp); Pam3Cys-Ser-(Lys)-4-3HCl (P3C); poly I:C (pI:C); regulatory T cell (Treg); transgenic $(\mathrm{Tg})$; tryptophan (trp)

Conflict of interest: The authors have declared that no conflict of interest exists.

Citation for this article: J. Clin. Invest. 114:270-279 (2004)

doi:10.1172/JCI200421275.
Toll-like receptors (TLRs) are expressed mainly on macrophages and DCs and recognize signature microbial products. Although the interaction of TLRs with their microbial ligands activates innate immunity to mount a defense mechanism, i.e., upregulation of costimulatory molecules and the production of chemokines and cytokines (11), it also elicits a counter-regulatory response. In particular, others and we have demonstrated that bacterial DNA, its synthetic immunostimulatory sequence oligodeoxynucleotide (ISS-ODN) analogs (TLR9 ligands), and LPS (TLR4 ligand) induce indoleamine 2,3-dioxygenase (IDO) expression in vivo and in vitro $(12,13)$.

IDO, the rate-limiting tryptophan-catabolizing (trp-catabolizing) enzyme, is induced by IFNs (14) and enhanced by IL-10 but is suppressed by IL-4 and IL-13 (15). IDO is expressed in various cell types, including fibroblasts, macrophages, DCs, trophoblasts (14), and epithelial cells (16). DCs expressing IDO contribute to the generation and maintenance of peripheral tolerance by depleting autoreactive T cells (17-19) and by inducing Treg responses (20). The IDO-specific inhibitor 1-methyl-DL-tryptophan (M-trp) reverses these effects $(20,21)$.

As IDO inhibits T cell reactivity (22) and induces a Treg response (20), we hypothesized that the induction of IDO by certain TLR ligands could provide the missing link between microbial exposure and the inhibition of allergic asthma and organ-specific autoimmunity. Our studies presented below indicate the inhibitory role of TLR ligand-induced IDO in both Th2-mediated and Th1-mediated lung inflammation.

\section{Results}

Differential induction of IDO by ISS-ODN in various organs. We initially evaluated IDO enzymatic activity in various organs after i.v. ISS-ODN administration. Increased IDO enzymatic activity was observed in the lung, small intestine, colon, uterus, and heart. In contrast, we did not detect a statistically significant increase in IDO activity in the spleen, kidney, pancreas, or brain (Table 1). The kinetics of IDO activity in the lung and the spleen are presented in Figure 1A. Although we did not detect an increase in IDO activity in spleen homogenates, when we fractionated 
Table 1

Differential induction of IDO by ISS-ODN in various organs

\begin{tabular}{lccc} 
& \multicolumn{3}{c}{ IDO activity } \\
KYN (ng/mg protein/30 min) \\
Organ & PBS & ISS-ODN & Fold increase \\
Lung & $325 \pm 15$ & $2,785 \pm 433^{A}$ & 8.8 \\
Spleen & $249 \pm 71$ & $271 \pm 15$ & 1.2 \\
Small intestine & $522 \pm 33$ & $2,072 \pm 100^{A}$ & 4.0 \\
Colon & $351 \pm 79$ & $3,135 \pm 559^{A}$ & 8.9 \\
Kidney & $249 \pm 27$ & $317 \pm 33$ & 1.3 \\
Pancreas & $235 \pm 37$ & $325 \pm 36$ & 1.4 \\
Uterus & $298 \pm 18$ & $808 \pm 50^{\mathrm{A}}$ & 2.7 \\
Heart & $246 \pm 8$ & $448 \pm 21^{\mathrm{A}}$ & 1.8 \\
Brain & $175 \pm 27$ & $160 \pm 20$ & 0.9 \\
\hline
\end{tabular}

BALB/c mice were injected i.v. with ISS-ODN (20 $\mu \mathrm{g} /$ mouse) or vehicle (PBS). Four days after injection, IDO enzymatic activity in the various tissue homogenates was determined by KYN assay. ${ }^{A} P \leq 0.05$ vs. PBStreated or mutated ODN-treated (M-ODN-treated) mice.

the splenocytes into subpopulations we clearly detected induction of IDO activity by ISS-ODN in CD $11 c^{+}$cells $(<1 \%$ of total splenocytes) (Table 2). The induction of IDO in the lungs was similarly provoked by subcutaneous, intraperitoneal, or intratracheal administration of ISS-ODN (Figure 1B). IDO expression in the lung tissue was detected in alveolar and bronchial epithelial cells as well as in cells with macrophage and/or DC features, as shown by immunohistochemical staining (Figure 1, C-J).

Induction of IDO activity is mediated by IFN- $\gamma$ and IL-12. ISS-ODN is known to induce TNF- $\alpha$, IL-6, IL-12, and IFNs in mice (23). To elucidate the role of ISS-ODN-induced cytokines in IDO expression, we analyzed IDO activity in TNF- $\alpha$, IL-6, IL-12p40, IFN- $\gamma$, IFN- $\alpha / \beta$ receptor (IFN- $\alpha / \beta$ R), or IL- $1 \beta$ receptor (IL- $1 \beta R$ ) $\mathrm{KO}$ mice after ISS-ODN administration. An induction of IDO enzymatic activity in the lungs was detected in TNF- $\alpha$, IL-6, IFN- $\alpha / \beta$ R, and IL- $1 \beta$ R KO mice but was absent in IL-12p40 and IFN- $\gamma$ KO mice (Table 3 and Figure $2 \mathrm{~A}$ ).

The above results indicate that IL-12p40 and/or IFN- $\gamma$ are necessary for IDO induction. The induction of IDO by IL-12 is mediated by IFN- $\gamma$, and IFN- $\gamma$ was shown to directly induce IDO expression in the lung tissue (24). In contrast to those observations, ISS-ODN induced IDO transcript in bone marrow-derived DCs (BMDCs) from IFN- $\gamma$ KO mice similar to that induced in BMDCs from WT mice (Figure 2B). Furthermore, lung CD11 $\mathrm{c}^{+}$cells isolated from IFN- $\gamma$ KO mice after in vivo ISS-ODN administration display a comparable level of IDO expression and enzymatic activity, as was detected in lung CD11 $\mathrm{c}^{+}$cells isolated from WT mice under the same conditions (Figure $2, \mathrm{C}$ and $\mathrm{D})(<1 \%$ of total pulmonary IDO activity). These data indicate that the induction of IDO in pulmonary DCs (CD11c cells) by ISS-ODN is independent of IFN- $\gamma$. In contrast, induction of high levels of IDO enzymatic activity in whole lung tissue, which is composed mainly of alveolar and bronchial epithelial cells $\left(\mathrm{CD} 11 \mathrm{c}^{+}\right)$, is dependent on IFN- $\gamma$.

We next assessed the relationship between low levels of pulmonary IDO activity and the ability of ISS-ODN to inhibit experimental asthma in OVA/alum-sensitized, OVA-challenged, ISSODN-treated IL-12p40 or IFN- $\gamma$ KO mice. These KO mice, unlike their WT controls, were resistant to ISS-ODN treatment and developed airway hyperreactivity (AHR; provocative concentration of methacholine $200\left[\mathrm{PC}_{200}\right]$, or the concentration of MCh needed to induce a 200\% increase of enhanced pause [Penh] from baseline measurements]) and eosinophilic inflammation (Table 4).

IDO inhibits experimental asthma. The data above could suggest that ISS-ODN-induced pulmonary IDO plays a role in the inhibition of experimental asthma. To validate this assumption, we administered the IDO inhibitor M-trp to OVA/alum-sensitized, ISS-ODN-treated WT (BALB/c) mice. M-trp implantation competitively inhibited in vivo IDO activity as assessed by kynurenine $(\mathrm{KYN})$ level in the lungs (Figure 3A). Furthermore, M-trp significantly reduced the effects of ISS-ODN on experimental asthma as shown by increases in AHR (measurements of \%Penh and airway resistance in Figure 3, B and C, respectively), eosinophilic infiltration (Figure 3D), and levels of IL-5 and IL-13 in the bronchoalveolar lavage (BAL) fluid (Table 5). In contrast, M-trp did not interfere with the Th2-mediated pulmonary inflammation (Figures 3 and 4 and Table 5), suggesting that the M-trp effects are not due to some nonspecific antiinflammatory activities of this compound.

IDO controls the pulmonary survival of an OVA-specific Th2 cell line in vivo. To explore whether the inhibition of experimental asthma by ISS-ODN is mediated by IDO-induced cell death of a Th2 cell line, we adoptively transferred an in vitro-differentiated, OVA-specific transgenic (Tg) Th2 cell line (DO11.10) to SCID mice that were treated with ISS-ODN, or with ISS-ODN and M-trp, and then challenged with intranasal OVA. Increased IDO activity was observed in the lung homogenates of ISS-ODN-treated SCID mice (Figure 4A). The administration of ISS-ODN, with or without M-trp, inhibited antigen-specific (OVA-specific) T cell trafficking into the lung (Figure 4B) but not into the spleen (Figure 4C). ISS-ODN administration enhanced cell death of this Th2 cell line in the lungs, and this enhancement was reversed by M-trp (Figure $4 \mathrm{D})$. The induction of cell death of the Th2 cell line was organ-specific, since no difference in cell death of the transferred $\mathrm{Tg}$ Th2 cell line was observed in the spleens of recipient mice (Figure 4E). Figure 4, D and E, represent a snapshot of the cell death seen on day 5 after ISS-ODN administration. In addition, the administration of ISS-ODN reduced the levels of Th2 cytokines (IL-4, IL-5, and IL-13) in the BAL fluid, and this reduction was partially reversed by the IDO inhibitor M-trp (Table 6).

IDO controls the pulmonary survival of an OVA-specific Th1 cell line but not of $B$ cells in vivo. IDO activity has already been demonstrated to inhibit activated Th1 cells via the induction of apoptosis $(14,25)$. Furthermore, IDO activity was recently shown to inhibit the rejection of pulmonary allografts by recipient $\mathrm{T}$ cells (26). To explore whether ISS-ODN-induced IDO also controls pulmonary Th1 cell survival, we adoptively transferred in vitro-differentiated, OVAspecific Tg Th1 cell line (DO11.10) or WT B cells to SCID mice (Figure 5). In contrast to the Th2 transfer, OVA challenge in these mice significantly increased IDO activity in the lungs, and the administration of ISS-ODN further enhanced this activity (Figure 5A). The in vivo administration of ISS-ODN did not further increase the direct KYN measurement in the lung homogenate; this reflects that the actual in vivo KYN levels were determined by the in vivo trp availability (Figure 5B). As expected, M-trp implantation significantly reduced in vivo KYN levels in the lungs (Figure 5B). Neutrophil infiltration in the BAL fluid, an indicator of a Th1 lung inflammation (27), was significantly enhanced by M-trp implantation (Figure 5C). In addition, OVA challenge significantly enhanced cell death of the Th1 cell line in the lung (Figure 5D), but not in the spleen (Figure 5E), and the implantation of M-trp reversed this effect (Figure 5D). In contrast, the survival of B cells 
A

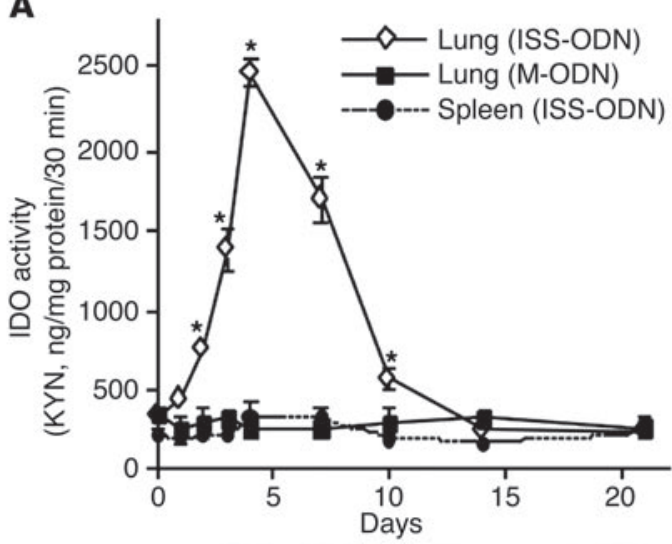

B

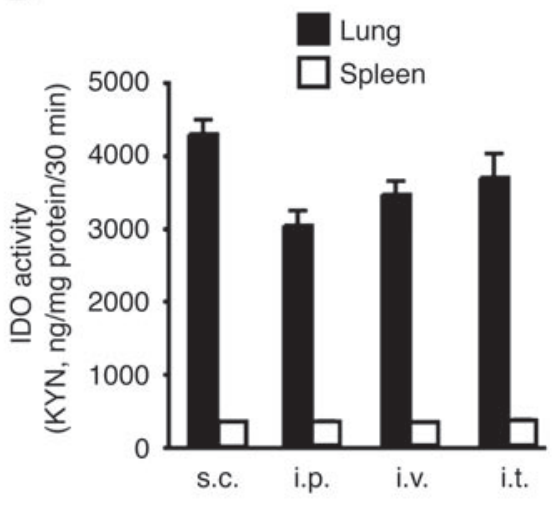

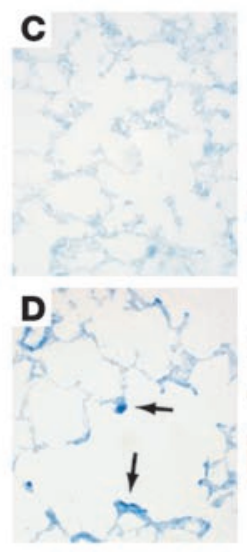

E
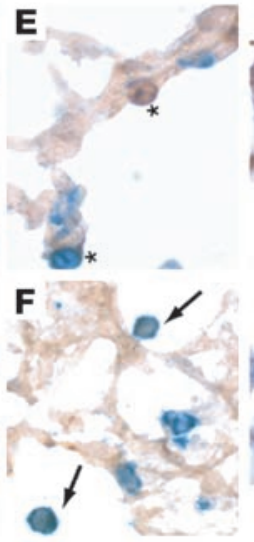
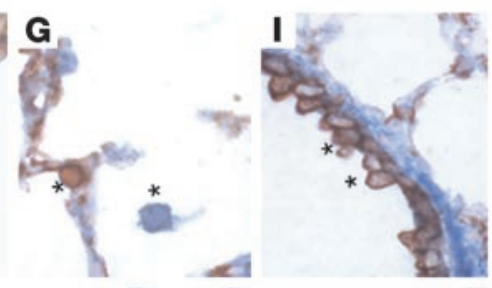

H

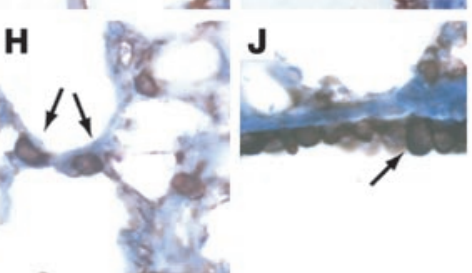

Figure 1

Differential induction of IDO by ISS-ODN. (A) BALB/c mice were injected i.v. with ISS-ODN $(20 \mu \mathrm{g})$ or mutated ODN (M-ODN) (20 $\mu \mathrm{g})$, and the lungs and spleen were harvested on days 1, 2, 3, 4, 7, 10, 14, and 21. The kinetics of IDO enzymatic activity in the lung and spleen homogenates were determined (KYN assay) and plotted. ${ }^{*} P \leq 0.05$ vs. PBS-treated or M-ODN-treated mice. (B) ISS-ODN (20 $\left.\mu \mathrm{g} / \mathrm{mouse}\right)$ was delivered to $\mathrm{BALB} / \mathrm{c}$ mice via different routes: subcutaneously (s.c.), intraperitoneally (i.p.), i.v., or intratracheally (i.t.). Four days later, IDO enzymatic activity in the lung and spleen homogenates was assessed (KYN assay). Data are means \pm SEM of results from four mice per group and represent one of three independent experiments that yielded similar results. (C-J) Immunohistochemistry of lung tissue. Naive mice (C, E, G, and I) and mice that were injected with ISS-ODN (D, F, H, and $\mathbf{J}$ ) were sacrificed 4 days after injection. (C and $\mathbf{D})$ Staining with rabbit anti-IDO Ab. Arrows indicate IDO-positive cells (D). (E and F) Staining with F4/80 and anti-IDO Ab. *F4/80-positive single cell (blue) or IDO-positive single cell (brown) (E). Arrows indicate double-positive cells (F). (G-J) Staining with anti-keratin Ab and anti-IDO Ab. *Anti-keratin-positive single cell (brown) or antiIDO-positive single cell (blue) (G and I). Arrows indicate double-positive cells (H and J). Original magnification: C, $\times 100 ; \mathbf{D}, \times 200 ; \mathbf{E}-\mathbf{J}, \times 400$.

transferred to SCID mice was not affected in the lungs (Figure $5 \mathrm{~F}$ ) or in the spleen (Figure 5G) after OVA challenge and ISSODN administration. Similar data were obtained in experiments designed to evaluate $\mathrm{B}$ cell death in an antigen-specific model using OVA-immunized BALB/c mice (data not shown).

Induction of IDO and inhibition of experimental asthma by certain $T L R$ ligands. Inhibition of experimental asthma has been documented for ISS-ODN (28) and for high but not for low doses of LPS (29). To determine the relationship between IDO induction by various microbial TLR ligands and the inhibition of experimental asthma, we first measured pulmonary IDO activity after administration of TLR ligands. As shown in Figure 6, only ISSODN and high-dose LPS $(50 \mu \mathrm{g})$ induced IDO enzymatic activity in the lungs. However, the high dose of LPS was associated with $70 \%$ mortality. We attributed this effect to LPS toxicity mainly in the lung tissue. IDO enzymatic activity correlated with reduced AHR (i.e., increased $\mathrm{PC}_{200}$ ) and with reduced eosinophil infiltration in the BAL fluid (Table 7).

\section{Discussion}

Our results indicate that the induction of pulmonary IDO by ISSODN protects the lung from Th2-driven lung inflammation and experimental asthma. Likewise, the induction of IDO in the SCID/ Th1 transfer model attenuated Th1-driven lung inflammation.

Table 2

Induction of IDO by ISS-ODN in splenic CDD11c+ cells

\begin{tabular}{lccc}
\hline & \multicolumn{3}{c}{ IDO activity } \\
& \multicolumn{3}{c}{ KYN (ng/mg protein/30 min) } \\
Cells & PBS & ISS-ODN & Fold increase \\
Spleen CD11C & $622 \pm 124$ & $1,985 \pm 23^{\mathrm{A}}$ & 3.2 \\
Spleen CD11 & $391 \pm 150$ & $379 \pm 16$ & 1.0
\end{tabular}

Splenic CD11 $\mathrm{C}^{+}$and CD11 $\mathrm{C}^{-}$cells were isolated at day 4 after ISSODN injection, and IDO enzymatic activity was determined by KYN assay. ${ }^{A} P \leq 0.05$ vs. PBS-treated or M-ODN-treated mice. 
Table 3

Induction of IDO in lungs by ISS-ODN in various cytokine KO mice

\begin{tabular}{|c|c|c|c|}
\hline \multirow[b]{2}{*}{ Treatment } & \multicolumn{3}{|c|}{$\begin{array}{c}\text { IDO activity } \\
\text { KYN (ng/mg protein/30 min) }\end{array}$} \\
\hline & PBS & ISS-ODN & Fold increase \\
\hline $\mathrm{BALB} / \mathrm{c}^{\mathrm{A}}$ & $380 \pm 55$ & $2,827 \pm 104^{D}$ & 7.4 \\
\hline C57BL/6B & $235 \pm 32$ & $2,582 \pm 78^{D}$ & 10.9 \\
\hline 129SvEvC & $309 \pm 30$ & $3,887 \pm 95^{\mathrm{D}}$ & 13 \\
\hline IFN- $\gamma \mathrm{KO}$ & $354 \pm 86$ & $205 \pm 14$ & 0.6 \\
\hline IL-12p40 K0 & $335 \pm 48$ & $136 \pm 41$ & 0.4 \\
\hline IFN- $\alpha / \beta R$ KO & $297 \pm 68$ & $1,531 \pm 90^{\mathrm{D}}$ & 5.4 \\
\hline TNF- $\alpha$ KO & $469 \pm 151$ & $3,256 \pm 450^{D}$ & 6.9 \\
\hline IL-6 KO & $272 \pm 32$ & $2,615 \pm 97^{D}$ & 9.6 \\
\hline IL-1 $\beta$ R KO & $179 \pm 45$ & $2,011 \pm 73^{D}$ & 11 \\
\hline
\end{tabular}

ISS-ODN (20 $\mu \mathrm{g} /$ mouse) was injected i.v. into IL-6, IFN- $\gamma$, IL-12p40, IFN- $\alpha / \beta R$, TNF- $\alpha$, and IL-1 $\beta R$ KO mice and their WT controls. The lungs were harvested 4 days after injection, and IDO enzymatic activity was measured (KYN assay). Data are means \pm SEM of three mice per group. AWT for IL-12p40 and IFN- $\gamma$ KO; BWT for TNF- $\alpha$, IL-6, and IL-1 $\beta R$ KO; ${ }^{C W T}$ for IFN- $\alpha / \beta R$ KO. DP $<0.05$, ISS-ODN-treated vs. PBS-treated group.

However, in this case, in contrast to the Th2 transfer model, the inhibition of lung inflammation by ISS-ODN administration was buffered by the intrinsic ability of Th1 cells to induce pulmonary IDO activity after OVA challenge, most probably via the production of IFN- $\gamma$, a potent transcriptional activator of IDO (24). In fact, our data suggest that the induction of pulmonary IDO by antigen-specific Th1 cell lines negatively controls their own survival and their inflammatory response, i.e., M-trp implantation reduced Th1 cell death (Figure 5D) and increased neutrophil infiltration, a marker of pulmonary inflammation (Figure 5C).

IDO can inhibit Th-mediated lung inflammation in multiple ways: (a) it depletes trp availability in the microenvironment (22); (b) it promotes the generation of various toxic trp metabolites, which induce Th cell death $(30,31)$; (c) it generates other compounds, e.g., formylkynurenine, through a reaction that removes oxygen radicals at inflammatory sites (32); and (d) in the case of Th2-mediated lung inflammation, it inhibits the generation of 5-hydroxytryptamine, a potent airway constrictor (33). These unique and synergistic activities of IDO likely collaborate to inhibit Th2-mediated experimental asthma and to attenuate Th1-mediated pulmonary inflammation. These effects were organ-specific and cell type-specific, since IDO did not affect the survival of adoptively transferred $\mathrm{Tg}$ Th cell lines in the spleen (Figures 4 and 5) and did not alter B cell survival in the lung or the spleen (Figure 5). The cell death seen in Figure 4, D and E, and Figure 5, D-G, represents only a fraction of the total cell death resulting from ISS-ODN-induced IDO, since these numbers reflect only a snapshot of the cell death seen on day 5 after ISS-ODN administration.

The induction of IDO by ISS-ODN in BMDCs and lung $\mathrm{CD} 11 \mathrm{c}^{+}$cells did not depend on IFN- $\gamma$ (Figure 2 ). In contrast, the high level of IDO enzymatic activity observed in the lung tissue (i.e., epithelial cells) (Figure 1) after ISSODN administration was dependent on IFN- $\gamma$ (Figure 2). Indeed, in other studies we confirmed that IFN- $\gamma$ induced IDO expression and IDO enzymatic activity in the murine respiratory epithelial cell line MLE-15 (data not shown). This high enzymatic activity of IDO in the lung epithelial cells rather than in the lung DCs appears necessary to inhibit the Th2-mediated lung inflammation (Figures 3 and 4, Tables 4 and 5). A similar mechanism has been observed in the placenta (34), where high tissue levels of IDO inhibit maternal T cell responses against the developing fetus (21).

Our results also indicate that activation of innate immunity by ISS-ODN, via the induction of IDO, suppresses lung inflammation in the adoptive transfer models. These models rule out the generation of $\mathrm{CD} 4{ }^{+} \mathrm{CD} 25^{+}$Treg or of $\operatorname{Tr} 1$ cells, thereby eliminating their contribution to the inhibition of lung inflammation via cognate interaction or via their secretion of IL-10 (35). The transfer models, therefore, establish the fundamental contribution of activated innate immunity to the inhibition of Th2- and Th1-mediated lung inflammation. We have previously demonstrated that this protective effect is transient (36) and therefore may require a periodic exposure to immunostimulatory DNA (ISS-DNA; i.e., bacterial DNA) in order to maintain the inhibition of allergic phenotype in a sensitized host. However, epidemiological evidence indicates that the exposure to microbial agents in early childhood provides long-term protection against allergic asthma, suggesting that there is an induction of a memory, adaptive immune response. Treg cells were recently proposed to mediate this protection (10). In this respect, recent studies have also demonstrated a crucial role for IDO-producing DCs in the induction of a Treg response (19). Thus, the exposure to certain microbial agents or to their signature compounds (e.g., TLR ligands) can provide an immediate and/or long-term, IDO-related protective effect against Th2- and Th1-driven inflammation. The

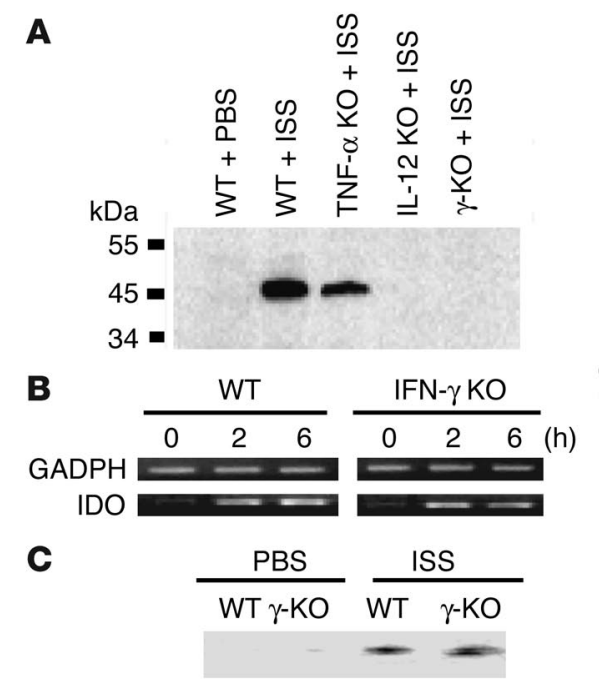

Figure 2

The impact of ISS-ODN-induced cytokines on IDO expression levels. (A) Western blot of lung homogenates (40 $\mu$ g protein) from TNF- $\alpha$, IL-12p40, and IFN- $\gamma$ KO mice and WT mice that were injected i.v. with ISS-ODN $(20 \mu \mathrm{g})$. (B-D) Lung CD11c ${ }^{+}$ cells were isolated from WT or IFN- $\gamma \mathrm{KO}(\gamma-\mathrm{KO})$ mice (five mice per group) that were injected i.v. with ISS-ODN (ISS) (20 $\mu \mathrm{g} /$ mouse). IDO expression was assessed by RT-PCR (B), Western blot (C), and measurements of enzymatic activity (KYN assay) (D). ${ }^{*} P \leq 0.05$, ISS-ODN-treated vs. PBS-treated group. 
Table 4

Inhibition of experimental asthma by ISS-ODN in IFN- and IL-12 K0 mice

\begin{tabular}{lcccc}
\hline & \multicolumn{2}{c}{ PC $_{\text {200 }}$} & \multicolumn{2}{c}{$\%$ Eosinophils } \\
Treatment & PBS & ISS-0DN & PBS & ISS-ODN \\
BALB/C & $3.0 \pm 0.4$ & $9.4 \pm 2.6^{A}$ & $68 \pm 4$ & $32 \pm 5^{A}$ \\
IFN- $\gamma$ K0 & $2.2 \pm 1$ & $2.3 \pm 0.5$ & $67 \pm 4$ & $62 \pm 12$ \\
IL-12p40 K0 & $2.6 \pm 0.6$ & $1.9 \pm 1.1$ & $68 \pm 7$ & $69 \pm 5$ \\
\hline
\end{tabular}

IFN- $\gamma$ KO or IL-12p40 KO mice were subcutaneously immunized with OVA/alum (days 0 and 7) and challenged with OVA twice (days 16 and 21) (four mice per group). ISS-ODN (50 $\mu \mathrm{g} /$ mouse) was given intraperitoneally 1 day before OVA-inhalation challenge (day 16). AHR $\left(\mathrm{PC}_{200}\right)$ and eosinophilic infiltration with and without ISS-ODN treatment were compared. Naive mice and mice immunized with OVA and challenged with $\mathrm{PBS}$ showed a $\mathrm{PC}_{200}$ of $9.4 \pm 0.8$ and less than $1 \%$ eosinophil infiltration. Data shown are means \pm SEM of four mice per group and represent one of two independent experiments that yielded similar results. ${ }^{A} P \leq 0.05$, ISS-ODN-treated vs. PBS-treated group.

immediate protective effect is mediated by innate immunity; is organ-specific, i.e., in the lung, placenta, and most probably other organs, as shown in Table 1; and is driven by high levels of IDO enzymatic activity in nonimmune cells. This high level of IDO activity generates a hostile and cytopathic microenvironment for the infiltrating Th cells. In contrast, the long-term protective effect induced by microbial agents (including helminths) or their signature compounds is mediated by adaptive immunity (10). It is initiated by low levels of IDO enzymatic activity in DCs, which elicit effector and memory Treg responses that counter-regulate proinflammatory $\mathrm{T}$ cell responses.

Our results also provide insight into recent data documenting the differential effects of LPS on the induction of experimental asthma. While administration of low doses of LPS did not prevent the induction of experimental asthma, administration of high doses inhibited its development (29). As is presented here, high doses but not low doses of LPS induced pulmonary IDO enzymatic activity. Likewise, other TLR ligands, such as Pam3Cys-Ser(Lys)-4-3HCl (P3C; TLR2 ligand), poly I:C (pI:C; TLR3 ligand), and flagellin (Flag; TLR5 ligand), did not induce pulmonary IDO activity and did not provide an immediate protection against experimental asthma (Figure 6 and Table 7).

Why do the lungs have a special ability to produce high levels of IDO upon stimulation with microbial products such as bacterial DNA? A potential answer is based on the unique physiological role of the lung. This organ has evolved to maintain its gas-exchange function while interacting with airborne microorganisms. The lung can develop strong, organ-specific antimicrobial activity
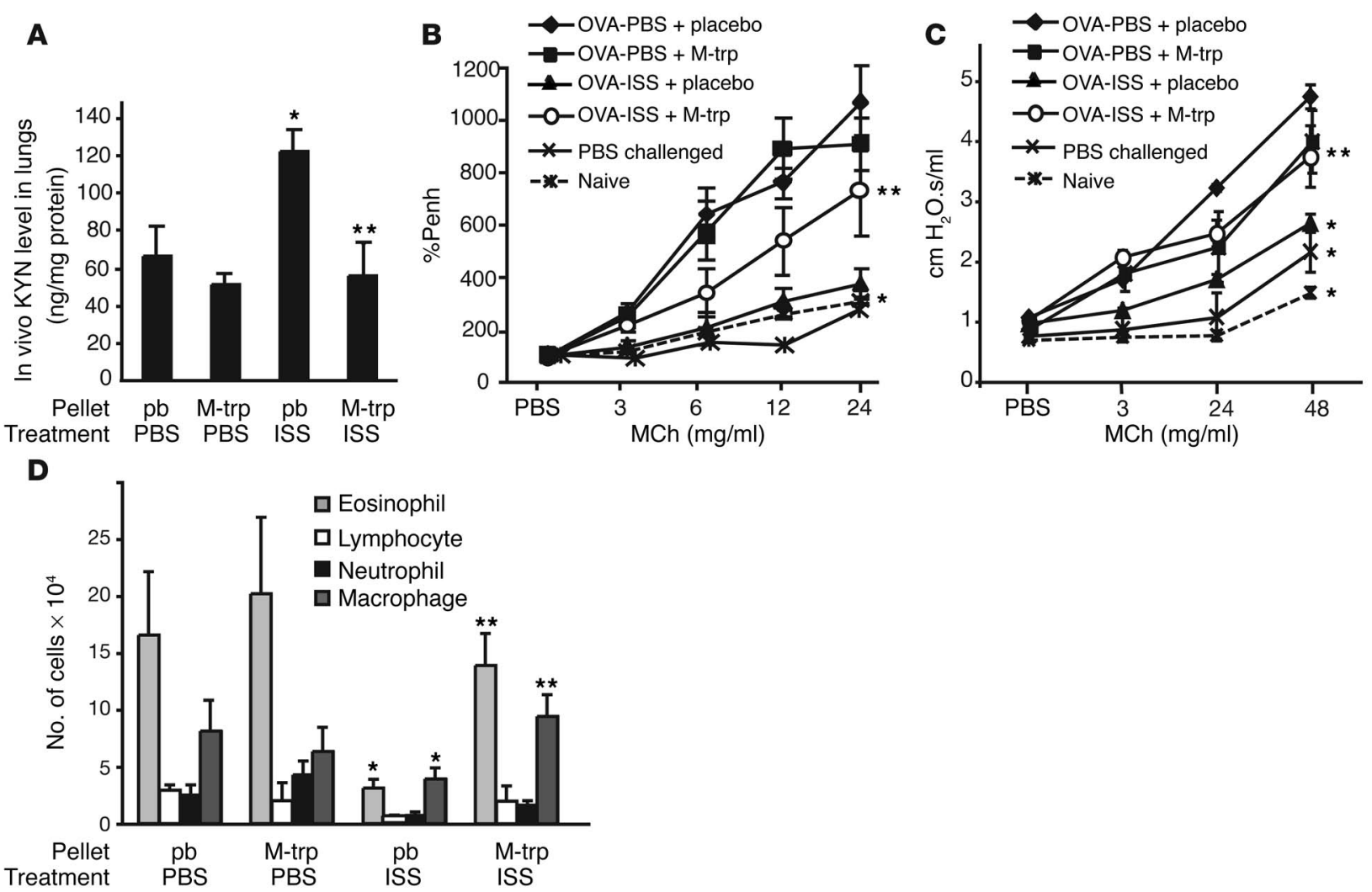

Figure 3

Reversal of ISS-ODN-induced inhibition of experimental asthma by M-trp. BALB/c mice (five mice per group) were sensitized by subcutaneous OVA/alum on day 0 and day 7 . On days 16 and 21, mice were challenged intranasally with $5 \mu \mathrm{g}$ OVA. ISS-ODN (50 $\mu \mathrm{g})$ was given intraperitoneally on day 16. AHR was measured on day 22. M-trp or placebo $(\mathrm{pb})$ pellets were implanted (day 15). Naive mice and OVA-immunized, PBS-challenged mice served as controls. (A) In vivo KYN levels in lung homogenates. (B) AHR measurements (\%Penh). (C) Measurement of airway resistance. (D) Total cell number of eosinophils, lymphocytes, neutrophils, and macrophages in the BAL fluid. ${ }^{*} P \leq 0.05$, ISS-ODN-treated (50 $\mu \mathrm{g} /$ mouse) vs. PBS-treated group. ${ }^{* *} P \leq 0.05$, ISS-ODN-treated mice with M-trp pellets vs. ISS-ODN-treated mice with placebo (pb) pellets. 
Table 5

Th2 cytokine levels in the BAL fluid in experimental asthma

\begin{tabular}{lcc} 
Treatment & IL-5 & IL-13 (pg/ml) \\
PBS + placebo & $206 \pm 28$ & $127 \pm 42$ \\
PBS + M-trp & $223 \pm 48$ & $128 \pm 37$ \\
ISS-ODN + placebo & $60 \pm 32^{\mathrm{A}}$ & $<15^{\mathrm{A}}$ \\
ISS-ODN + M-trp & $149 \pm 25^{\mathrm{B}}$ & $60 \pm 0.3^{\mathrm{B}}$ \\
\hline
\end{tabular}

Data shown are means \pm SEM and represent of four independent experiments that yielded similar results. ${ }^{A} P \leq 0.05$, ISS-ODN-treated ( $50 \mu \mathrm{g} /$ mouse) vs. PBS-treated group. ${ }^{\mathrm{B}} P \leq 0.05$, ISS-ODN-treated mice with M-trp pellets vs. ISS-ODN-treated mice with placebo $(\mathrm{pb})$ pellets.

while at the same time limiting the potential damage inflicted on the lung parenchyma by subsequent inflammatory processes. IDO has antimicrobial properties against a variety of intracellular pathogens, such as parasites (e.g., Toxoplasma gondii) (37), bacteria (e.g., Listeria monocytogenes) (38), mycobacteria (e.g., Mycobacterium avium) (12), and viruses (e.g., cytomegalovirus) (39). The inhibition of intracellular growth of these microorganisms can occur (a) by depletion of intracellular levels of the rarest amino acid, trp, which is essential for their growth, and/or (b) by the generation of antimicrobial trp metabolites. Interestingly, by the very same mechanisms, IDO inhibits Th cell-mediated inflammatory responses and therefore limits the potential damage to lung function. A similar rationale can be applied to IDO induction in the small intestine and the colon (Table 1).
In summary, the induction of IDO by certain microbial TLR ligands results in immediate and long-term immunoregulatory activities. In certain aspects this induction of IDO provides a biochemical and an immunological basis for the hygiene and the counter-regulatory hypotheses. However, additional pathways of innate immunity are activated by other microbial products and could promote immunoregulatory functions in the host. One such innate pathway is via DC-SIGN, a C-type lectin expressed on DCs (40), which, upon ligation with viral, bacterial, mycobacterial, fungal, or helminth-derived carbohydrate structures, induced the secretion of IL-10, an immunosuppressive cytokine, which also promotes a Treg cell response (41). Thus, the activation of other pathways of innate immunity may also contribute to the inverse association between microbial exposure and the prevalence of allergic and autoimmune diseases. The identification of such novel microbial products allows for the application to modern medicine of lessons initially learned through the hygiene hypothesis.

\section{Methods}

Animals. Mice were purchased from The Jackson Laboratory (Bar Harbor, Maine, USA). IFN- $\alpha / \beta$ R KO mice were purchased from B\&K Universal Ltd. (East Yorkshire, United Kingdom). All animal procedures followed the University of California, San Diego, animal-care guidelines.

Reagents. ISS-ODN (5'-TGACTGTGAACGTTCGAGATGA-3') and mutated ODN (M-ODN) (5'-TGACTGTGAAGGTTAGAGATGA-3') were purchased from Trilink Biotechnologies (San Diego, California, USA). P3C was purchased from EMC Microcol-
Figure 4

IDO induces cell death of a Th2 cell line and inhibits experimental asthma in an adoptively transferred SCID mouse model. SCID mice (six mice per group) were implanted with M-trp pellets, adoptively transferred with $5 \times 10^{6}$ cells of a Tg Th2 cell line (DO11.10) per mouse, challenged with OVA $(50 \mu \mathrm{g})$, and treated with ISS-ODN (50 $\mu \mathrm{g} / \mathrm{mouse})$. (A) IDO enzymatic activity in the lung homogenates. (B and $\mathbf{C}$ ) The numbers of adoptively transferred Th2 cells in the lungs (B) and spleens (C) were calculated by staining with anti-CD4 and anti-T cell receptor Ab (KJ 1-26; FACS). (D and E) The percentage of cell death of the Th2 cell line in lungs (D) and in spleens (E) was determined by staining with 7-AAD (FACS). The total number of cells in the organ was calculated by multiplication of the total number of live cells by the percentage of CD4+/DO11.10 $\mathrm{TCR}^{+}$ cells in the cell suspension. The percentage of $7-A A D+$ cells was determined in the CD4+/DO11.10 $\mathrm{TCR}^{+}$gate (30). ${ }^{*} P \leq 0.05$, OVA-challenged, PBS-treated mice (with placebo and M-trp pellets) vs. nonchallenged group. ${ }^{* *} P<0.05$, ISSODN-treated mice with placebo pellets vs. PBS-treated mice with placebo pellets. ${ }^{* * *} P \leq 0.05$, ISS-ODN-treated mice with M-trp pellets vs. ISS-ODN-treated mice with placebo pellets.
A

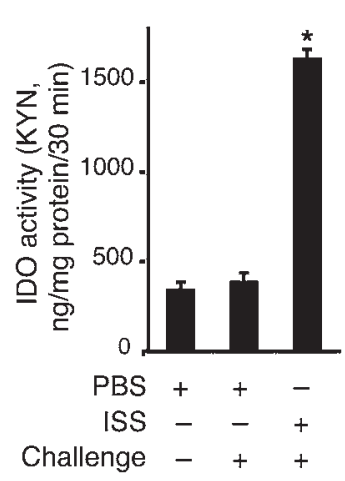

\section{B}

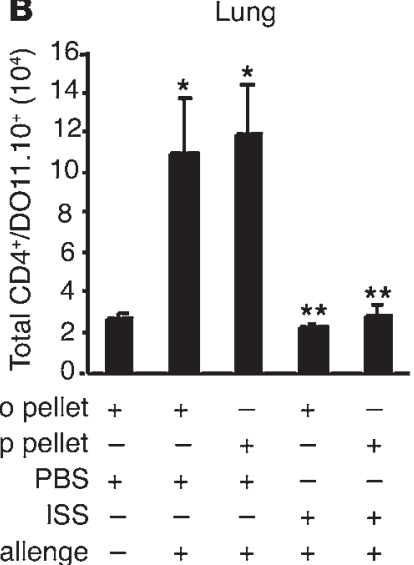

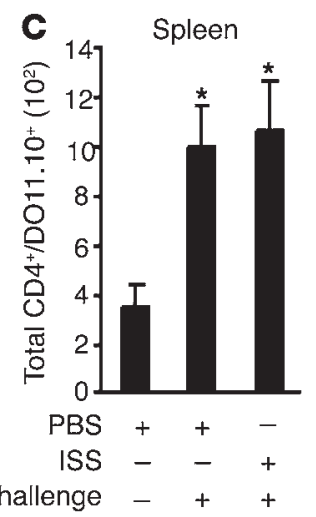

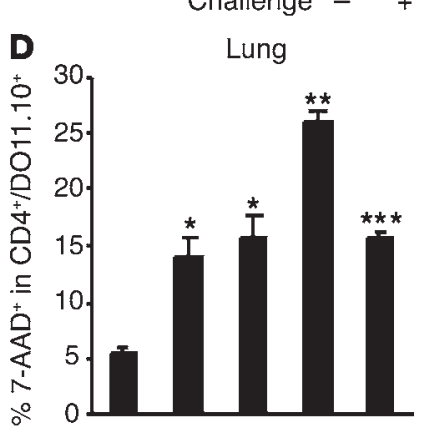

Placebo pellet $+\quad+\quad-\quad+-$

M-trp pellet $-\quad-++$

PBS +++-

ISS - $-\quad++$

Challenge -++++ 
Table 6

Th2 cytokine levels in the BAL fluid of adoptively transferred SCID mice

$\begin{array}{lcccc}\text { Challenge } & \text { Treatment } & \text { IL-4 } & \text { IL-5 } & \text { IL-13 (pg/ml) } \\ - & \text { PBS + placebo } & 36 \pm 8.8 & <15 & 67 \pm 5.8 \\ + & \text { PBS + placebo } & 393 \pm 83^{\mathrm{A}} & 320 \pm 80^{\mathrm{A}} & 262 \pm 50^{\mathrm{A}} \\ + & \text { ISS-ODN + placebo } & 84 \pm 0.5^{\mathrm{B}} & 74 \pm 20^{\mathrm{B}} & 113 \pm 3.3^{\mathrm{B}} \\ + & \text { ISS-ODN + M-trp } & 196 \pm 35^{\mathrm{C}} & 168 \pm 12^{\mathrm{C}} & 244 \pm 71^{\mathrm{C}}\end{array}$

Data are means \pm SEM of one of three independent experiments that yielded similar results. ${ }^{A} P \leq 0.05$, OVA-challenged, PBS-treated mice (with placebo and $\mathrm{M}$-trp pellets) vs. nonchallenged group. ${ }^{\mathrm{B}} P<0.05$, ISS-ODN-treated mice with placebo pellets vs. PBS-treated mice with placebo pellets. ${ }^{C} P \leq 0.05$, ISS-ODN-treated mice with M-trp pellets vs. ISS-ODN-treated mice with placebo pellets.

lections GmbH (Tübingen, Germany), Flag was kindly provided by J. DiDonato (Cleveland Clinic Foundation, Cleveland, Ohio, USA) (42), and LPS and PI:C were purchased from Sigma-Aldrich (St. Louis, Missouri, USA). OVA and various trp metabolites were purchased from Sigma-Aldrich.
Measurement of IDO $m R N A$ levels and enzymatic activity. IDO mRNA levels were measured by RT-PCR using the sense primer $5^{\prime}$-TTATGCAGACTGTGTCYTGGCAACTG- $3^{\prime}$ and the antisense primer 5'-TTTCCAGCCAGACARATATATGCGRAG-3', as previously described (12). We used two measurements to evaluate IDO activity. First, maximal IDO activity was indirectly determined by incubation of various organ homogenates with an excess of exogenous $\operatorname{trp}(780 \mu \mathrm{M})$ as a substrate in the reaction buffer for 30 minutes in vitro (43). The resulting KYN levels in the reaction homogenates were measured colorimetrically, and the IDO activity was expressed as nanograms KYN per milligrams protein per 30 minutes (43). Second, to determine the impact of the IDO-competitive inhibitor $\mathrm{M}$-trp on IDO activity in vivo, we directly assessed KYN levels in lung homogenates. The resulting IDO activity in these organs was expressed as nanograms KYN per milligrams protein. Protein concentrations were determined using a BCA protein assay kit (Pierce Chemical Co., Rockford, Illinois, USA).

Culture of BMDCs and isolation of pulmonary and splenic CD11 $c^{+} D C$. BMDCs were prepared from BALB/c or IFN- $\gamma$ KO mice as previously described (42). Single-cell suspensions of lung or spleen were prepared by collagenase digestion as previously described (44). The DC population was initially enriched by discontinuous
A
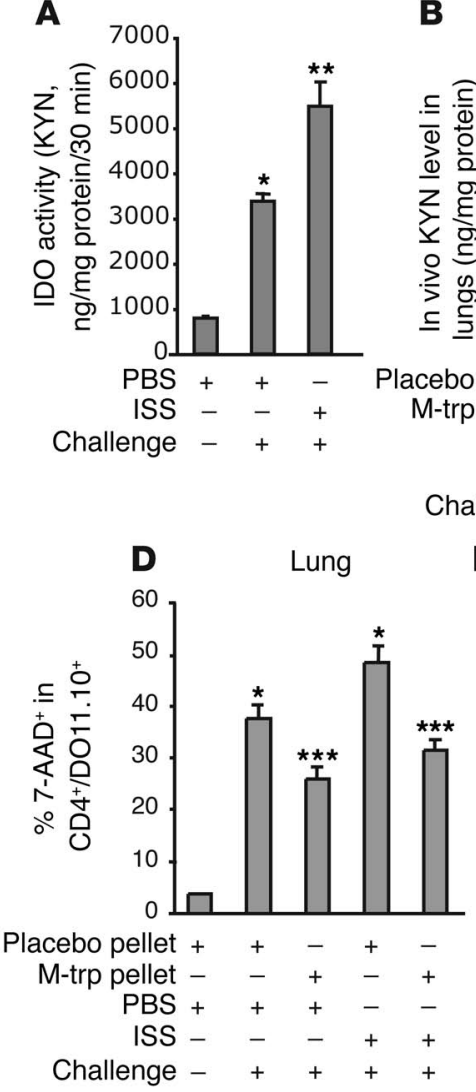

B

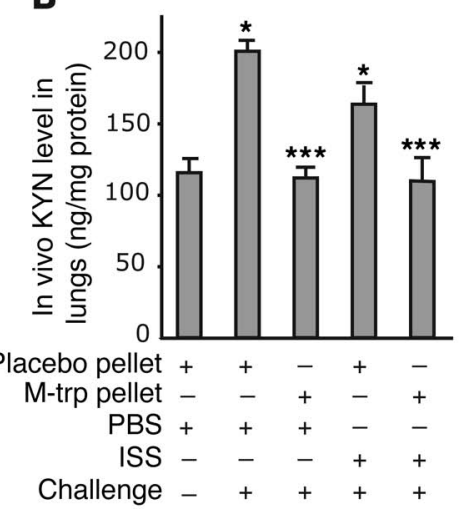

E

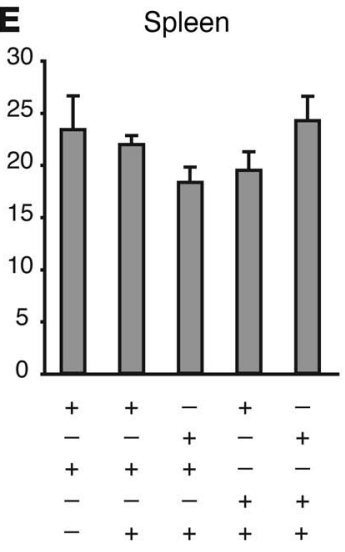

C
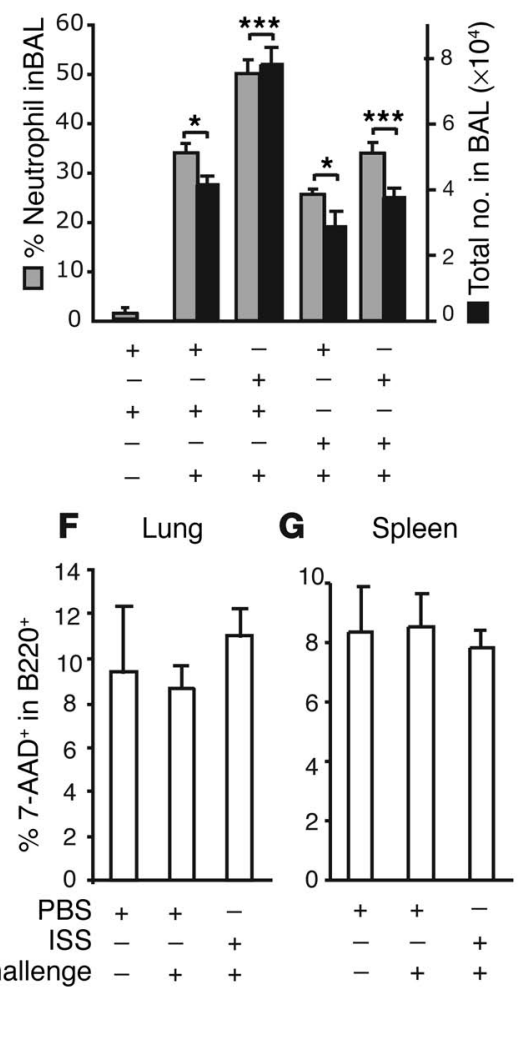

Figure 5

IDO induces cell death of a Th1 cell line, but not B cell death, in the lungs of adoptively transferred SCID mice. SCID mice (six mice per group) were implanted with M-trp pellets, adoptively transferred with $5 \times 10^{6}$ cells of a Tg Th1 cells line (DO11.10) per mouse, challenged with OVA $(50 \mu \mathrm{g})$, and treated with ISS-ODN (50 $\mu \mathrm{g} /$ mouse). (A and B) IDO activity (A) and in vivo KYN levels (B) in the lung homogenates. (C) Neutrophil infiltrates in BAL fluid. (D and E) The percentage of cell death of the Th1 line in lungs (D) and in spleens (E) was assessed. ${ }^{\star} P<0.05$ vs. nonchallenged group. ${ }^{* *} P<0.05$, PBS-treated vs. ISS-ODN-treated mice. ${ }^{* *} P<0.05$, mice with M-trp pellets vs. mice with placebo pellets. ( $F$ and $\mathbf{G})$ B cells $\left(5 \times 10^{6}\right.$ per mouse) were transferred i.v. to SCID mice treated with ISS-ODN or PBS and challenged with OVA for 4 days (six mice per group). The percentage of $B$ cell death in lungs $(\mathbf{F})$ and in spleens $(\mathbf{G})$ was determined by 7-AAD staining. 


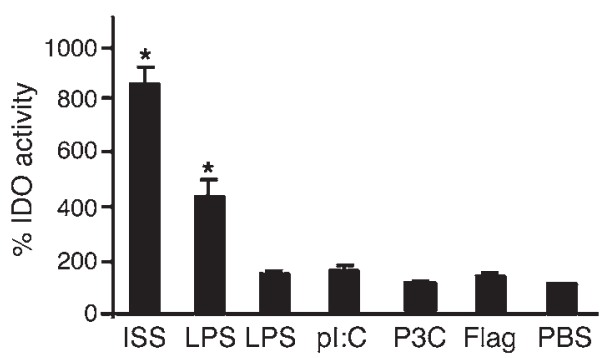

Figure 6

Induction of IDO by various TLR ligands. BALB/c mice (four mice per group) were injected i.v. with ISS-ODN $(20 \mu \mathrm{g})$, LPS (50 or $1 \mu \mathrm{g})$, poly I:C (pl:C, $20 \mu \mathrm{g})$, Pam3Cys-Ser-(Lys)-4-3HCl (P3C, $25 \mu \mathrm{g})$, or flagellin (Flag, $50 \mu \mathrm{g}$ ). IDO activity in lung homogenates was assessed 4 days after injection by KYN assay. IDO activity is expressed as fold induction over the activity measured in PBS-injected mice (100\%). ${ }^{*} P \leq 0.05$ vs. OVA-immunized and PBS-treated mice.

density gradient centrifugation using OptiPrep (Axis-Shield PoC AS, Oslo, Norway) according to the manufacturer's instruction. Low-density cells in the interface were harvested, and CD $11 c^{+}$ cells were positively isolated using anti-CD11c magnetic beads (Miltenyi Biotec Inc., Auburn, California, USA). The CD11 ${ }^{+}$population was greater than $90 \%$ of the total cells as determined by FACS analysis. High-density cells were collected and served as a CD11c cell population.

Experimental asthma. Airway inflammation was induced by a protocol previously described (45) with minor modifications. In brief, mice (BALB/c, IFN- $\gamma$ KO, and IL-12 KO) were sensitized by subcutaneous injection of $20 \mu \mathrm{g}$ OVA absorbed with $500 \mu \mathrm{g}$ alum (Alhydrogel; Brenntag Biosector AS, Federikssund, Denmark) per mouse in saline on day 0 and day 7 . On days 16 and 21, mice were challenged intranasally with $5 \mu \mathrm{g}$ OVA per mouse. In some groups, ISS-ODN $(50 \mu \mathrm{g} / \mathrm{mouse})$ or one of various TLR ligands was given intraperitoneally 1 day before OVA challenge (day 16). Twenty-four hours after the last challenge (day 22), mice were subjected to measurements of airway responsiveness and sacrificed by $\mathrm{CO}_{2}$ asphyxiation. In experiments using $\mathrm{M}$-trp, pellets containing either M-trp (80 mg, 10 mg/d release; Innovative Research of America, Sarasota, Florida, USA) or placebo were surgically implanted under the dorsal skin 1 day before ISS-ODN treatment (day 15). Naive mice and OVA/alum-sensitized, PBS-challenged mice served as controls.

After the mice were sacrificed, BAL cells, lungs, and spleen were recovered. The total number of cells in BAL were counted, and BAL cells were stained with Wright-Giemsa stain for the differential count. Percentages of eosinophils, lymphocytes, neutrophils, and macrophages were determined microscopically using standard morphological criteria (36). The total cell number of each cell type was calculated from the total cell number and the percentage of each cell type in the differential cell count. The level of each cytokine (IL-4, IL-5, and IL-13) in the BAL fluid was determined by ELISA (BD Biosciences - Pharmingen, San Diego, California, USA).

Table 7
Airway responsiveness to methacholine ( $\mathrm{MCh}$ ) was assessed 24 hours after the final OVA challenge using a single-chamber, whole-body plethysmograph obtained from Buxco Electronics Inc. (Wilmington, North Carolina, USA), as previously described (45). The Penh, a dimensionless value, which correlates well with pulmonary resistance measured by conventional two-chamber plethysmography in ventilated mice (45), was used to monitor airway responsiveness. In the plethysmograph, mice were exposed for 3 minutes to nebulized PBS to establish base-line Penh values and were subsequently exposed to increasing concentrations of nebulized MCh (Sigma-Aldrich) in PBS using an Aerosonic ultrasonic nebulizer (DeVilbiss Health Care Inc., Somerset, Pennsylvania, USA). Following each nebulization, recordings were taken for 3 minutes. The Penh values measured during each 3-minute sequence were averaged and are expressed for each MCh concentration as the percentage of base-line Penh values following PBS exposure (45). The $\mathrm{PC}_{200}$ concentration of MCh describes the provocative concentration of $\mathrm{MCh}$ that induced a $200 \%$ increase of Penh from base-line measurements (36).

To determine airway resistance, mice were anesthetized and a midcervical skin incision was made to expose the trachea, which was cannulated with an 18-gauge blunt needle. The airway resistance was measured with an in-line pressure transducer and software program (SCIREQ Inc., Montreal, Quebec, Canada) in intubated and ventilated mice connected to a rodent ventilator, as previously described (46). Airway resistance measurements were taken at base line, after nebulized PBS, and after each increasing concentration of nebulized MCh $(46,47)$.

Preparation of OVA-specific Th1 and Th2 cell lines. CD4 ${ }^{+} \mathrm{T}$ cells were positively selected from splenocytes of Tg DO11.10 mice using a MACS CD4 ${ }^{+} \mathrm{T}$ cell isolation kit (Miltenyi Biotec Inc.). Naive CD4 ${ }^{+} \mathrm{T}$ cells $\left(5 \times 10^{5}\right)$ were cultured with $5 \times 10^{6}$ irradiated splenocytes in RPMI 1640 supplemented with $0.3 \mu \mathrm{M}$ OVAspecific peptide $\left(\mathrm{OVA}_{323-339}\right)$ for 7 days (48). For in vitro Th1 differentiation, recombinant IL-12 $(40 \mathrm{ng} / \mathrm{ml}$; BD Biosciences Pharmingen) and neutralizing anti-IL-4 mAb (11B11, $10 \mu \mathrm{g} / \mathrm{ml}$; $\mathrm{BD}$ Biosciences - Pharmingen) were added to the culture media. For in vitro Th2 differentiation, recombinant IL-4 (25 ng/ml; BD Biosciences - Pharmingen) and anti-IL-12 mAb (C17.8, $10 \mu \mathrm{g} / \mathrm{ml}$;

Inhibition of experimental asthma by TLR ligands

\begin{tabular}{lcccccc} 
& & \multicolumn{5}{c}{ Total cell number in BAL $\left(\times \mathbf{1 0}^{6}\right)$} \\
Treatment & Challenge & $\mathbf{P C}_{200}$ & Eosinophil & Lymphocyte & Neutrophil & Macrophage \\
PBSA & PBS & $8.9 \pm 0.6^{\mathrm{B}}$ & $<0.5^{\mathrm{B}}$ & $<0.5^{\mathrm{B}}$ & $<0.5^{\mathrm{B}}$ & $<0.5^{\mathrm{B}}$ \\
PBS & OVA & $2.6 \pm 0.6$ & $23 \pm 5$ & $3 \pm 1$ & $2.3 \pm 0.5$ & $1.8 \pm 0.4$ \\
ISS-ODN & OVA & $9.7 \pm 2.6^{\mathrm{B}}$ & $1.6 \pm 0.3^{\mathrm{B}}$ & $1.0 \pm 0.2$ & $1.4 \pm 0.3$ & $2.1 \pm 0.4$ \\
LPS $(50 \mu \mathrm{g})$ & OVA & $6.3 \pm 1.5^{\mathrm{B}}$ & $7.6 \pm 2.3^{\mathrm{B}}$ & $3.6 \pm 1.1$ & $5.5 \pm 1.7^{\mathrm{B}}$ & $9.1 \pm 2.8^{\mathrm{B}}$ \\
LPS $(1 \mu \mathrm{g})$ & OVA & $3.6 \pm 0.4$ & $35 \pm 10$ & $5.2 \pm 1.6$ & $2.0 \pm 0.6$ & $5.0 \pm 2.0$ \\
pl:C & OVA & $5.9 \pm 2.1$ & $27 \pm 7$ & $8.2 \pm 3.5$ & $2.8 \pm 0.5$ & $4.7 \pm 1.4^{\mathrm{B}}$ \\
P3C & OVA & $2.7 \pm 1.1$ & $40 \pm 9$ & $9.5 \pm 2.6^{\mathrm{B}}$ & $3.0 \pm 0.8$ & $1.7 \pm 0.7$ \\
Flag & OVA & $2.6 \pm 1.6$ & $30 \pm 7$ & $2.8 \pm 0.7$ & $0.3 \pm 0.1$ & $4.1 \pm 1.0^{\mathrm{B}}$
\end{tabular}

OVA/alum-sensitized BALB/c mice (four mice per group) were treated with ISS-ODN (50 $\mu \mathrm{g}$ ), LPS $(50 \mu \mathrm{g}$ or $1 \mu \mathrm{g})$, poly I:C (pl:C, $50 \mu \mathrm{g})$, Pam3Cys-Ser-(Lys)-4-3HCl (P3C, $25 \mu \mathrm{g}$ ), or flagellin (Flag, $50 \mu \mathrm{g}$ ) intraperitoneally prior to OVA challenge. OVA/alum-immunized, PBS-challenged mice served as controls. AHR ( $\left.\mathrm{PC}_{200}\right)$ and the total number of eosinophils, lymphocytes, neutrophils, and macrophages in the BAL fluid were determined. Data shown are means \pm SEM of three independent experiments. Mortality of $70 \%$ was observed in the group treated with LPS $(50 \mu \mathrm{g})$, whereas no mortality was detected in the other groups. ${ }^{A}$ Mice immunized with OVA and challenged with PBS served as controls. ${ }^{\mathrm{B} P} \leq 0.05$ vs. OVA-immunized and PBS-treated mice. 
BD Biosciences - Pharmingen) were added to the culture media. The commitment to a Th1 or Th2 phenotype was confirmed by CFSE labeling of the $\mathrm{T}$ cells after the initial OVA stimulation (more than five cell divisions as determined by FACS) and by measurement of their cytokine profile in the supernatants after OVA stimulation (49): in vitro-differentiated Th1 cell lines secreted high IFN- $\gamma(2.83 \pm 0.09 \mathrm{ng} / \mathrm{ml})$ and undetectable IL-4, IL-5, and IL-13 $(<50 \mathrm{pg} / \mathrm{ml})$, whereas in vitro-differentiated Th2 cell lines secreted high IL-4 (79.5 $\pm 7.7 \mathrm{ng} / \mathrm{ml})$, IL-5 $(10.7 \pm 1.0 \mathrm{ng} / \mathrm{ml})$, and IL-13 $(21.7 \pm 1.7 \mathrm{ng} / \mathrm{ml})$ and undetectable IFN- $\gamma(<50 \mathrm{pg} / \mathrm{ml})$.

Adoptive transfers and induction of Th1- and Th2-mediated lung inflammation. In vitro-differentiated OVA-specific Th1 or Th2 cell lines $\left(5 \times 10^{6}\right)$ were transferred i.v. to SCID mice on day 0 as previously described (48). Mice that received the Th2 cell line were given intranasal OVA $(50 \mu \mathrm{g})$ daily for 4 days (days 1-4) before being analyzed, whereas mice that received the Th 1 cell line were given intranasal OVA $(50 \mu \mathrm{g})$ twice, once on day 3 and once on day 4. On day 5, mice were sacrificed, BAL fluid was collected, and the lungs were perfused with PBS and harvested for further studies. BAL cells were Wright-Giemsa-stained (36), and single-cell suspensions of spleen and lung were prepared by being passed through a sterile cell strainer.

In some experiments, B cells were isolated from the spleens of $\mathrm{BALB} / \mathrm{c}$ mice using MACS CD19 microbeads (Miltenyi Biotec Inc.), and $5 \times 10^{6}$ purified $B$ cells were then transferred i.v. into SCID mice treated with or without ISS-ODN and then intranasally challenged with OVA as described for Th2 transfer. To study antigen-specific B cell death, BALB/c mice were immunized four times, on days $0,7,14$, and 21 , with OVA $(20 \mu \mathrm{g})$ absorbed with alum $(500 \mu \mathrm{g})$. Mice were challenged with OVA twice, once on day 26 and once on day 31. Mice were then sacrificed 24 hours after the last challenge. Lung cells and splenocytes were prepared and stained for FACS analysis (see below).

FACS analysis, Western blot, and bistological examination. Cells were stained for CD4 (BD Biosciences - Pharmingen) and DO11.10 T cell receptor (Caltag Laboratories, Burlingame, California, USA). B cells were identified with anti-B220 Ab (BD Biosciences - Pharmingen). Staining with 7-amino-actinomycin $\mathrm{D}$ (7-AAD; BD Biosciences - Pharmingen) was performed accord- ing to the manufacturer's instructions. The stained cells were analyzed using a FACSCalibur flow cytometer (BD Biosciences - Immunocytometry Systems, San Jose, California, USA). Western blot was performed using rabbit anti-IDO antiserum (43) following standard methods.

Ten-micrometer cryosectioned lung tissues were stained with anti-F4/80 (Serotec Inc., Raleigh, North Carolina, USA) followed by biotin anti-rat $\mathrm{Ab}$ (BD Biosciences - Pharmingen), and visualized by alkaline phosphatase-streptavidin (Jackson ImmunoResearch Laboratories Inc., West Grove, Pennsylvania, USA) and Vector Blue (Vector Laboratories Inc., Burlingame, California, USA). These sections were subsequently stained with rabbit anti-IDO Ab (50) followed by HRP-anti-rabbit Ab (Jackson ImmunoResearch Laboratories Inc.) and then developed with NovaRed (Vector Laboratories Inc.). Sections were also stained with rabbit anti-keratin $\mathrm{Ab}$ (DakoCytomation California Inc., Carpinteria, California, USA) followed by DAKO EnVision System HRP-anti-rabbit Ab (Dakocytomation California Inc.) and then visualized with NovaRed. These sections were subsequently stained with anti-IDO Ab, followed by alkaline phosphatase-anti-rabbit Ab, and then developed with Vector Blue (Vector Laboratories Inc.).

Statistics. Data were compared using the Student's $t$ test. A $P$ value of less than or equal to 0.05 was considered statistically significant.

\section{Acknowledgments}

We thank Nissi Varki (University of California, San Diego) for histological evaluation and Robert Coffman (Dynavax Technologies Corp.) for helpful comments. This work was supported by NIH grants AI40682, DK35108, AR47360 (to E. Raz), and AI56453 (to D. Carson). E. Raz is supported by a grant from Dynavax Technologies Corp.

Received for publication February 9, 2004, and accepted in revised form May 18, 2004.

Address correspondence to: Eyal Raz, University of California, San Diego, Department of Medicine, 9500 Gilman Drive, La Jolla, California 92093-0663, USA. Phone: (858) 534-5444; Fax: (858) 534-5399; E-mail: eraz@ucsd.edu.
1. Bach, J.F. 2002. The effect of infections on susceptibility to autoimmune and allergic diseases. N. Engl. J. Med. 347:911-920.

2. Strachan, D.P. 1989. Hay fever, hygiene, and household size. BMJ. 299:1259-1260.

3. Howarth, P.H. 1998. Is allergy increasing? Early life influences. Clin. Exp. Allergy. 28(Suppl. 6):2-7.

4. Martinez, F.D., and Holt, P.G. 1999. Role of microbial burden in aetiology of allergy and asthma. Lancet. 354(Suppl. 2):SII12-SII15.

5. Weiss, S.T. 2002. Eat dirt: the hygiene hypothesis and allergic diseases. N. Engl. J. Med. 347:930-931.

6. Kemp, A., and Bjorksten, B. 2003. Immune deviation and the hygiene hypothesis: a review of the epidemiological evidence. Pediatr. Allergy Immunol. 14:74-80.

7. Shaheen, S.O., et al. 1996. Measles and atopy in Guinea-Bissau. Lancet. 347:1792-1796.

8. Shirakawa, T., Enomoto, T., Shimazu, S., and Hopkin, J.M. 1997. The inverse association between tuberculin responses and atopic disorder. Science. 275:77-79.

9. Godfrey, D.G. 1978. Identification of economically important parasites. Nature. 273:600-604.

10. Wills-Karp, M., Santeliz, J., and Karp, C.L. 2001. The germless theory of allergic disease: revisit- ing the hygiene hypothesis. Nat. Rev. Immunol. 1:69-75.

11. Takeda, K., Kaisho, T., and Akira, S. 2003. Toll-like receptors. Annu. Rev. Immunol. 21:335-376.

12. Hayashi, T., et al. 2001. Enhancement of innate immunity against Mycobacterium avium infection by immunostimulatory DNA is mediated by indoleamine 2,3-dioxygenase. Infect. Immun. 69:6156-6164.

13. Hissong, B.D., Byrne, G.I., Padilla, M.L., and Carlin, J.M. 1995. Upregulation of interferoninduced indoleamine 2,3-dioxygenase in human macrophage cultures by lipopolysaccharide, muramyl tripeptide, and interleukin-1. Cell. Immunol. 160:264-269

14. Mellor, A.L., and Munn, D.H. 2001. Extinguishing maternal immune responses during pregnancy: implications for immunosuppression. Semin. Immunol. 13:213-218.

15. Grohmann, U., et al. 2002. CTLA-4-Ig regulates tryptophan catabolism in vivo. Nat. Immunol. 3:1097-1101.

16. Babcock, T.A., and Carlin, J.M. 2000. Transcriptional activation of indoleamine dioxygenase by interleukin 1 and tumor necrosis factor alpha in interferontreated epithelial cells. Cytokine. 12:588-594.
17. Hwu, P., et al. 2000. Indoleamine 2,3-dioxygenase production by human dendritic cells results in the inhibition of $\mathrm{T}$ cell proliferation. J. Immunol. 164:3596-3599.

18. Munn, D.H., et al. 2002. Potential regulatory function of human dendritic cells expressing indoleamine 2,3-dioxygenase. Science. 297:1867-1870.

19. Mellor, A.L., et al. 2003. Induced indoleamine 2,3 dioxygenase expression in dendritic cell subsets suppresses T cell clonal expansion. J. Immunol. 171:1652-1655.

20. Fallarino, F., et al. 2003. Modulation of tryptophan catabolism by regulatory $\mathrm{T}$ cells. Nat. Immunol. 4:1206-1212.

21. Munn, D.H., et al. 1998. Prevention of allogeneic fetal rejection by tryptophan catabolism. Science. 281:1191-1193.

22. Munn, D.H., et al. 1999. Inhibition of T cell proliferation by macrophage tryptophan catabolism. J. Exp. Med. 189:1363-1372.

23. Roman, M., et al. 1997. Immunostimulatory DNA sequences function as T helper-1-promoting adjuvants. Nat. Med. 3:849-854.

24. Takikawa, O., Tagawa, Y., Iwakura, Y., Yoshida, R., and Truscott, R.J. 1999. Interferon-gamma-dependent/independent expression of indoleamine 
2,3-dioxygenase. Studies with interferon-gammaknockout mice. Adv. Exp. Med. Biol. 467:553-557.

25. Fallarino, F., et al. 2002. T cell apoptosis by tryptophan catabolism. Cell Death Differ. 9:1069-1077.

26. Swanson, K.A., Zheng, Y., Heidler, K.M., Mizobuchi, T., and Wilkes, D.S. 2003. CD11 c $^{+}$cells modulate pulmonary immune responses by production of indoleamine 2,3-dioxygenase. Am. J. Respir. Cell Mol. Biol. 30:311-318.

27. Lloyd, C.M., et al. 2000. CC chemokine receptor (CCR)3/eotaxin is followed by CCR4/monocytederived chemokine in mediating pulmonary $\mathrm{T}$ helper lymphocyte type 2 recruitment after serial antigen challenge in vivo. J. Exp. Med. 191:265-274.

28. Horner, A.A., Van Uden, J.H., Zubeldia, J.M. Broide, D., and Raz, E. 2001. DNA-based immunotherapeutics for the treatment of allergic disease. Immunol. Rev. 179:102-118.

29. Eisenbarth, S.C., et al. 2002. Lipopolysaccharideenhanced, toll-like receptor 4-dependent $\mathrm{T}$ helper cell type 2 responses to inhaled antigen. J. Exp. Med. 196:1645-1651.

30. Terness, P., et al. 2002. Inhibition of allogeneic T cell proliferation by indoleamine 2,3-dioxygenase-expressing dendritic cells: mediation of suppression by tryptophan metabolites. J. Exp. Med. 196:447-457.

31. Frumento, G., et al. 2002. Tryptophan-derived catabolites are responsible for inhibition of $\mathrm{T}$ and natural killer cell proliferation induced by indoleamine 2,3-dioxygenase. J. Exp. Med. 196:459-468.

32. Caucheteux, S.M., Kanellopoulos-Langevin, C., and Ojcius, D.M. 2003. At the innate frontiers between mother and fetus: linking abortion with complement activation. Immunity. 18:169-172.

33. Barnes, P.J. 2001. Histamine and serotonin. Pulm.
Pharmacol. Ther. 14:329-339.

34. Sedlmayr, P., et al. 2002. Localization of indoleamine 2,3-dioxygenase in human female reproductive organs and the placenta. Mol. Hum. Reprod. 8:385-391.

35. Curotto de Lafaille, M.A., and Lafaille, J.J. 2002. $\mathrm{CD} 4(+)$ regulatory $\mathrm{T}$ cells in autoimmunity and allergy. Curr. Opin. Immunol. 14:771-778.

36. Broide, D.H., et al. 2001. Systemic administration of immunostimulatory DNA sequences mediates reversible inhibition of Th2 responses in a mouse model of asthma. J. Clin. Immunol. 21:175-182.

37. Daubener, W., et al. 2001. Restriction of Toxoplasma gondii growth in human brain microvascular endothelial cells by activation of indoleamine 2,3dioxygenase. Infect. Immun. 69:6527-6531.

38. Mackler, A.M., Barber, E.M., Takikawa, O., and Pollard, J.W. 2003. Indoleamine 2,3-dioxygenase is regulated by IFN- $\gamma$ in the mouse placenta during Listeria monocytogenes infection. J. Immunol. 170:823-830

39. Bodaghi, B., et al. 1999. Role of IFN- $\gamma$-induced indoleamine 2,3 dioxygenase and inducible nitric oxide synthase in the replication of human cytomegalovirus in retinal pigment epithelial cells. J. Immunol. 162:957-964.

40. van Kooyk, Y., and Geijtenbeek, T.B. 2003. DCSIGN: escape mechanism for pathogens. Nat. Rev. Immunol. 3:697-709.

41. Wakkach, A., et al. 2003. Characterization of dendritic cells that induce tolerance and $\mathrm{T}$ regulatory 1 cell differentiation in vivo. Immunity. 18:605-617.

42. Datta, S.K., et al. 2003. A subset of Toll-like receptor ligands induces cross-presentation by bone marrow-derived dendritic cells. J. Immunol.
170:4102-4110

43. Takikawa, O., Kuroiwa, T., Yamazaki, F., and Kido, R. 1988. Mechanism of interferon-gamma action. Characterization of indoleamine 2,3-dioxygenase in cultured human cells induced by interferongamma and evaluation of the enzyme-mediated tryptophan degradation in its anticellular activity. J. Biol. Chem. 263:2041-2048.

44. Vermaelen, K.Y., Carro-Muino, I., Lambrecht, B.N., and Pauwels, R.A. 2001. Specific migratory dendritic cells rapidly transport antigen from the airways to the thoracic lymph nodes. J. Exp. Med. 193:51-60.

45. Broide, D., et al. 1998. Immunostimulatory DNA sequences inhibit IL-5, eosinophilic inflammation, and airway hyperresponsiveness in mice. J. Immunol. 161:7054-7062.

46. Ikeda, R.K., et al. 2003. Resolution of airway inflammation following ovalbumin inhalation: comparison of ISS DNA and corticosteroids. Am. J. Respir. Cell Mol. Biol. 28:655-663.

47. Cho, J.Y., et al. 2004. Immunostimulatory DNA inhibits transforming growth factor- $\beta$ expression and airway remodeling. Am. J. Respir. Cell Mol. Biol. 30:651-661.

48. Hansen, G., Berry, G., DeKruyff, R.H., and Umetsu, D.T. 1999. Allergen-specific Th1 cells fail to counterbalance Th2 cell-induced airway hyperreactivity but cause severe airway inflammation. J. Clin. Invest. 103:175-183.

49. Grogan, J.L., and Locksley, R.M. 2002. T helper cell differentiation: on again, off again. Curr. Opin. Immunol. 14:366-372.

50. Suzuki, S., et al. 2001. Expression of indoleamine 2,3-dioxygenase and tryptophan 2,3-dioxygenase in early concepti. Biochem. J. 355:425-429. 